REVIEWS OF INFECTIOUS DISEASES - VOL. 4, SUPPLEMENT - NOVEMBER-DECEMBER 1982

(C) 1982 by The University of Chicago. All rights reserved. $0162-0886 / 82 / 0406-0017 \$ 02.00$

\title{
Comparative Multiple-Dose Pharmacokinetics of Cefotaxime, Moxalactam, and Ceftazidime
}

\author{
Ruedi Lüthy, Jürg Blaser, Antonio Bonetti, \\ Hanspeter Simmen, Richard Wise, and \\ Walter Siegenthaler
}

The present study was conducted to compare the pharmacokinetics of three different doses of cefotaxime, moxalactam, and ceftazidime and to evaluate the influence of probenecid on the pharmacokinetic behavior of these three third-generation cephalosporins.

\section{Patients and Methods}

Six male volunteers received in a crossover fashion doses of $0.5,1.0$, and $2.0 \mathrm{~g}$ of each drug by infusion over a 5 -min interval. Doses of $1.0 \mathrm{~g}$ were repeated after administration of probenecid. For each dose, 17 blood samples per volunteer were drawn for documentation of the distribution and elimination phase of the cephalosporin. The urinary excretion was determined from five quantitative urine collections made over the 24 -hr period after administration of the test drug. Serum and urine concentrations were assayed by an agar diffusion method. The assay strain for cefotaxime was resistant to its desacetyl metabolite. The average coefficient of variation for interassay precision was $4.6 \% \pm 0.9 \%$. Serum samples for the 0.5- and 2.0-g doses of cefotaxime and moxalactam also were analyzed by a method of highpressure liquid chromatography (HPLC) that provided information on the behavior of the desacetyl metabolite of cefotaxime and the two naturally occurring epimers of moxalactam [1,2]. The pharmacokinetic parameters of a two-compartment open model were adapted to the experimental data with a nonlinear fitting program. For all statistical

This paper is an extended abstract of a paper published in Antimicrobial Agents and Chemotherapy 20:567-575, 1981.

Informed consent according to institutional policies was obtained from each study participant.

This study was supported in part by grants from Lilly Research Laboratories and Glaxo Group Research, Ltd.

Please address requests for reprints to Dr. R. Lüthy, Division of Infectious Diseases, Department of Medicine, University of Zürich, CH-8091 Zürich, Switzerland.
From the Division of Infectious Diseases, Department of Medicine, University of Zürich, Zürich, Switzerland; and the Department of Medical Microbiology, Dudley Road Hospital, Birmingham, England

evaluations the Wilcoxon matched pairs signed rank test was used. Probabilities of $2 \alpha \leqslant 0.05$ were considered significant.

\section{Results and Discussion}

The mean serum concentrations of cefotaxime, moxalactam, and ceftazidime at $10 \mathrm{~min}$ and 6,8 , and $12 \mathrm{hr}$ are presented in table 1 . Serum concentrations of moxalactam exceeded those of ceftazidime at all times and were distinctly higher than those of cefotaxime. At 8 and 12 hr the mean concentrations of cefotaxime were consistently $<0.5 \mathrm{mg} /$ liter, whereas those of ceftazidime and moxalactam were $\sim 5$-10 times higher. For facilitation of the comparison between the various doses and drugs, the area under the serum concentration-vs.-time curve (AUC) was normalized by dividing by the individual dose. Compared with the value for cefotaxime, the normalized AUC for moxalactam was three to four times higher and that for ceftazidime, two to three times higher. Probenecid increased the AUC for the 1.0-g dose of cefotaxime about twofold but did not affect those for moxalactam and ceftazidime significantly. Linear regression analysis of the dose $(x$ [in $\mathrm{g}])$ vs. normalized AUC ( $y$ [in $\mathrm{mg} \times \mathrm{hr} /$ liter]) yielded a slope for cefotaxime $(y=13.14 x+45.12)$ that was significantly different from zero $(P<0.001)$, a result that indicated a nonlinear increment in AUC for increasing doses. In contrast, slopes for moxalactam $(y=-19.36 x+240.09)$ and ceftazidime $(y=-14.59 x+168.51)$ did not differ significantly from zero.

The pharmacokinetic parameters of cefotaxime, moxalactam, and ceftazidime are summarized in table 2. Significant differences between the three compounds in the total volume of distribution were observed for the $0.5 \mathrm{-g}$ dose but not for the 1.0- and $2.0-\mathrm{g}$ doses. Intraindividual comparisons of the elimination $t / 2$ values, total body clearance rate, and renal clearance rate demonstrated 
Table 1. Mean serum concentrations of drug after infusions over a 5-min interval of cefotaxime, moxalactam, and ceftazidime in six male volunteers.

\begin{tabular}{|c|c|c|c|c|c|}
\hline \multirow[b]{2}{*}{ Dose, drug } & \multirow[b]{2}{*}{ Actual dose $(\mathrm{g})^{*}$} & \multicolumn{4}{|c|}{ Mean \pm sD serum level (mg/liter) } \\
\hline & & $0.17 \mathrm{hr}$ & $6 \mathrm{hr}$ & $8 \mathrm{hr}$ & $12 \mathrm{hr}$ \\
\hline \multicolumn{6}{|l|}{$0.5 \mathrm{~g}$} \\
\hline Cefotaxime & 0.58 & $37.8 \pm 7.1$ & $0.3^{\dagger}$ & $<0.1$ & $<0.1$ \\
\hline Moxalactam & 0.47 & $63.3 \pm 6.0$ & $4.2 \pm 1.7$ & $2.2 \pm 0.6$ & $0.7 \pm 0.2$ \\
\hline Ceftazidime & 0.48 & $49.9 \pm 8.0$ & $2.1 \pm 0.5$ & $1.0 \pm 0.3$ & $0.3 \pm 0.1$ \\
\hline \multicolumn{6}{|l|}{$1 \mathrm{~g}$} \\
\hline Cefotaxime & 1.06 & $80.8 \pm 14.1$ & $0.4 \pm 0.1$ & $<0.1$ & $<0.1$ \\
\hline Moxalactam & 0.99 & $120 \pm 12.4$ & $8.2 \pm 2.0$ & $4.7 \pm 1.0$ & $1.4 \pm 0.5$ \\
\hline Ceftazidime & 0.96 & $107 \pm 18.0$ & $4.4 \pm 1.4$ & $2.1 \pm 0.7$ & $0.5 \pm 0.3$ \\
\hline \multicolumn{6}{|l|}{$2 \mathrm{~g}$} \\
\hline Cefotaxime & 2.02 & $174 \pm 36.7$ & $0.9 \pm 0.5$ & $0.5 \ddagger$ & $<0.1$ \\
\hline Moxalactam & 1.87 & $210 \pm 30.8$ & $14.2 \pm 2.4$ & $8.0 \pm 1.8$ & $2.6 \pm 0.8$ \\
\hline Ceftazidime & 1.94 & $181 \pm 23.2$ & $6.6 \pm 1.5$ & $3.8 \pm 0.9$ & $1.1 \pm 0.5$ \\
\hline \multicolumn{6}{|c|}{$1 \mathrm{~g}+$ probenecid $\S$} \\
\hline Cefotaxime & 0.96 & $109 \pm 12.3$ & $1.4 \pm 0.7$ & $0.6 \|$ & $<0.1$ \\
\hline Moxalactam & 1.10 & $111 \pm 10.8$ & $10.0 \pm 1.7$ & $6.2 \pm 1.2$ & $2.7 \pm 0.8$ \\
\hline Ceftazidime & 0.97 & $98.9 \pm 12.5$ & $4.2 \pm 0.6$ & $2.1 \pm 0.5$ & $0.5 \pm 0.2$ \\
\hline
\end{tabular}

* Mean value of the doses administered to the six volunteers.

$\dagger$ The SD for three volunteers was 0.1 ; that for the other three was $<0.1$.

¥ The so for three volunteers was 0.4 ; that for the other three was $<0.1$.

$\S$ Probenecid was administered at a dosage of $0.5 \mathrm{~g}$ every $6 \mathrm{hr}$ on the day before testing and $1.0 \mathrm{~g}$ at $30 \mathrm{~min}$ before administration of the test drug.

$\|$ The SD for five volunteers was 0.4 ; that for the remaining volunteer was $<0.1$.

significant differences between these antibiotics. The $t / 1 / 2$ values, calculated from the $0.5-, 1.0-$, and $2.0 \mathrm{-g}$ doses, averaged $2.34,1.95$, and $1.16 \mathrm{hr}$ for moxalactam, ceftazidime, and cefotaxime, respectively. The 24-hr urinary recovery was highest for moxalactam $(75 \% \pm 4 \%)$, followed by ceftazidime $(68 \% \pm 11 \%)$ and cefotaxime $(53 \% \pm 6 \%)$. The total body and renal clearance rates of cefotaxime decreased significantly with an increase in the dose. In contrast to the findings for moxalactam and ceftazidime, the ratio of renal clearance rate to creatinine clearance rate for cefotaxime indicated considerable tubular secretion of the drug.

The influence of probenecid on serum concentration, $t \frac{1}{2}, A U C$, volume of distribution, and clearance rate was most obvious with cefotaxime. Saturation of tubular secretion led to serum concentrations with the $1.0 \mathrm{~g}$ dose of cefotaxime that at $2 \mathrm{hr}$ already were higher than those achieved with the $2.0-\mathrm{g}$ dose. The renal clearance rate of this drug decreased by almost $50 \%$ and the AUC doubled when probenecid was administered. This finding is in contrast to the findings for moxalactam and ceftazidime, where the marginal influence of this agent is of no practical significance.
The level of the desacetyl metabolite of cefotaxime, determined by HPLC, peaked $45 \mathrm{~min}$ after administration. The average levels of the metabolite were $2.7 \pm 1.0$ and $9.8 \pm 1.8 \mathrm{mg} /$ liter for the 0.5 - and $2.0 \mathrm{~g}$ dose, respectively, and the half lives for these doses were approximately twice those of cefotaxime $(1.9 \pm 0.7 \mathrm{hr}$ and $1.4 \pm 0.4 \mathrm{hr}$, respectively). After the $0.5-\mathrm{g}$ dose the AUC for the desacetyl metabolite was $31 \% \pm 12 \%$ of the AUC for cefotaxime. For the $2.0 \mathrm{~g}$ dose this proportion decreased to $18 \% \pm 2 \%$, a result suggesting that desacetylation may not follow first-order kinetics.

Freshly prepared solutions of moxalactam contain two epimers, designated $R(-)$ and $S(-)$, in approximately equal amounts. The serum protein binding of the $\mathrm{R}(-)$ epimer averages $53 \%$; that of the $S(-)$ epimer is $\sim 67 \%$ [3]. The antimicrobial activity of the former epimer is approximately double that of $S(-)$ [1].

Analysis of the two epimers revealed that their pharmacokinetic behaviors are different. At 10 min after iv administration, the ratio of the $R(-)$ to the $S(-)$ epimer was 0.84 ; it decreased rapidly to 0.5 at $5 \mathrm{hr}-$ i.e., there was twice as much of the $S(-)$ epimer as of the $R(-)$ epimer. At this time 


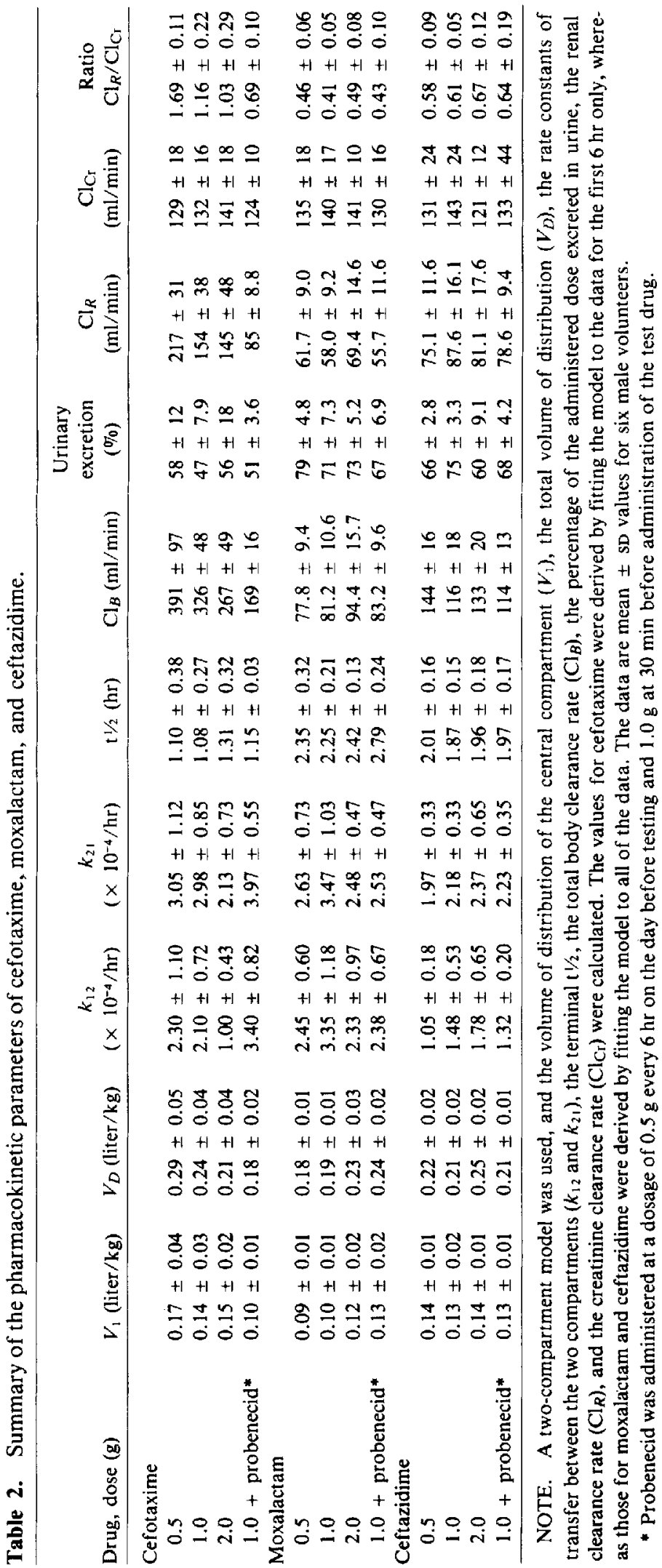


the antimicrobial activity, as determined by the agar diffusion assay, was reduced by one-fourth when compared with the concentration measured by HPLC analysis.

No adverse effects were recorded throughout the study. Chemistry profiles, blood cell counts, urinalysis results, and creatinine clearance rates remained within normal limits.

The present study demonstrated that significant differences exist in the pharmacokinetic behavior of cefotaxime, moxalactam, and ceftazidime. From this standpoint, it appears reasonable to conclude that moxalactam and possibly cef- tazidime could be administered twice a day and cefotaxime, three or even four times a day.

\section{References}

1. Wise, R., Wills, P. J., Bedford, K. A. Epimers of moxalactam: in vitro comparison of activity and stability. Antimicrob. Agents Chemother. 20:30-32, 1981.

2. Wise, R., Wright, N., Wills, P. J. Pharmacology of cefotaxime and its desacetyl metabolite in renal and hepatic disease. Antimicrob. Agents Chemother. 19:526-531, 1981.

3. Yamada, H., Ichihashi, T., Hirano, K., Kinoshita, H. Plasma protein binding and urinary excretion of $R(-)$ and $S$ (-) epimers of an arylmalonylamino 1-oxacephem. I. In humans. [letter]. J. Pharm. Sci. 70:112-113, 1981. 